This item was submitted to Loughborough's Research Repository by the author.

Items in Figshare are protected by copyright, with all rights reserved, unless otherwise indicated.

\title{
State and output-feedback shared-control for a class of linear constrained systems
}

PLEASE CITE THE PUBLISHED VERSION

https://doi.org/10.1109/tac.2015.2510158

PUBLISHER

(C) Institute of Electrical and Electronics Engineers (IEEE)

VERSION

AM (Accepted Manuscript)

LICENCE

CC BY-NC-ND 4.0

\section{REPOSITORY RECORD}

Jiang, Jingjing, and Alessandro Astolfi. 2015. "State and Output-feedback Shared-control for a Class of Linear Constrained Systems”. Loughborough University. https://hdl.handle.net/2134/36754. 


\title{
State and Output-Feedback Shared-Control for a Class of Linear Constrained Systems
}

\author{
Jingjing Jiang ${ }^{1}$ and Alessandro Astolfi ${ }^{2}$
}

\begin{abstract}
This paper presents state and output feedback sharedcontrol algorithms for a class of linear systems in the presence of constraints on the output described by means of linear inequalities. The properties of the closed-loop shared-control systems are studied using Lyapunov arguments. Simulation results demonstrate the effectiveness of the algorithm.
\end{abstract}

\section{INTRODUCTION}

Shared-control discussed in this paper refers to a control architecture in which there is a combination of a human operator input and a feedback control input. It has the same meaning as that described in [1] and as in the well-known anti-lock braking system. The human operator takes charge of the system in "normal" circumstance, while the feedback controller is active in case of emergencies, for example when the system evolves towards "dangerous" situations (to be formally defined). Typical applications could be found in vehicle design [2], tele-manipulation [3] and training systems [4]. The main objective of shared-control is to guarantee the "safety" of a given dynamical system. In the simplest scenario, safety is represented by a set of constraints on the state or the output of the system, which should be satisfied. The safety condition is therefore characterized by a sort of collision-free requirement. As a consequence, the safety goal can be regarded as an obstacle avoidance problem, see [5] for a similar problem in robotics. A widely used method to solve obstacle avoidance problems in robotics is the Virtual Field Histogram (VFH) method developed from the Virtual Force Field (VFF) method, a local path planning algorithm [6]. The VFH method utilizes a histogram grid, a statistical representation of the environment, to deal with sensor uncertainties. This method suffers from significant shortcomings similarly to the VFF method: existence of local minimizers and oscillatory motion (especially when passing through a narrow passage) [7]. Fuzzy control is often used in shared-control problems, see e.g. [8]. Note that even though systems with fuzzy control have a convenient user interface and are easy to design, there is no guarantee of the system performances. These drawbacks have been partly overcome by Lyapunov-like methods [9] and barrier Lyaponov functions [10]. However, due to the definition of barrier Lyapunov functions, the state of the system is not allowed to reach the boundary of the

\footnotetext{
${ }^{1} \mathrm{~J}$. Jiang is with the Department of Electrical and Electronic Engineering, Imperial College London, UK, E-mail: jingjing. jiang10@imperial.ac.uk

${ }^{2}$ A. Astolfi is with the Dept. of Electrical and Electronic Engineering, Imperial College London, London, SW7 2AZ, UK and DICII, University of Roma "Tor Vergata", Via del Politecnico 1, 00133 Rome, Italy, E-mail: a.astolfi@imperial.ac.uk
}

admissible space. Invariance control is an alternative way to address control problems with state constraints: e.g. in [11] the problem has been studied using feedback linearization and optimization tools.

Shared-control problems have also been studied in [12], in which a continuous scalar function used to guarantee the smooth transitions between the human input and the feedback control input has been given. In [13] and [14], the sharedcontrol problem has been studied using some tools from [15][17] for fully-actuated, linear, mechanical systems with statefeedback and output-feedback, respectively. Since the control authority is allocated between two controllers, the human and the feedback controller, the sharing scheme based on the hysteresis switch can achieve all requirements: it is a simple scheme and does not generate oscillations. This paper extends the results in [13] and [14], provides formal proofs of all technical statements, and includes additional theoretical results and simulation studies. The paper is organized as follows. Section II formulates the problem and provides basic definitions and assumptions. The main results are given in Section III and IV in which the shared-control algorithms with full state and partial state feedback are presented followed by the description of the formal properties of the closedloop system. Numerical examples to show the effectiveness of the proposed shared-control strategies are given in Section V. Finally, Section VI gives some conclusions and suggestions for future work.

Notation In this paper we mostly use standard notation. The non-standard definitions used in the paper are as follows. Let $\mathcal{S}(t)$ be, for any fixed $t$, a set, $B_{\epsilon}(x)$ be the ball centered at $x$ with radius $\epsilon>0, \overline{\mathcal{Q}}$ be the complement of the set $\mathcal{Q}$. Then $\lim _{t \rightarrow \infty} \mathcal{S}(t)$ denotes the limit, if it exists, of the set $\mathcal{S}(t)$ as $t \rightarrow \infty$. The limit equals $\mathcal{Q}$ if and only if $\forall \epsilon>0, \exists t_{\epsilon}>0$ such that $\mathcal{Q}^{\epsilon-} \subset \mathcal{S}(t) \subset \mathcal{Q}^{\epsilon+} \forall t \geq t_{\epsilon}$, where $\mathcal{Q}^{\epsilon+}=\left\{B_{\epsilon}(x), x \in\right.$ $Q\}, \mathcal{Q}^{\epsilon-}=\overline{\left((\overline{\mathcal{Q}})^{\epsilon+}\right)}$. Let $\mathcal{P}$ be a set and $\mathcal{P} \subseteq \mathbb{R}^{n}$. Then $S \mathcal{P}+T$ denotes the set defined as $\left\{x \in \mathbb{R}^{n} \mid x=S y+\right.$ $T, y \in \mathcal{P}\}$, where $S \in \mathbb{R}^{n \times n}$ and $T \in \mathbb{R}^{n}$. $\operatorname{col}(a, b)$ denotes a column vector obtained by stacking the vector $b$ under $a$. Let $x=\left[x_{1}, x_{2}, \ldots, x_{n}\right]^{T} \in \mathbb{R}^{n}$. Then $|x|$ denotes the vector defined as $|x|=\left[\left|x_{1}\right|,\left|x_{2}\right|, \ldots,\left|x_{n}\right|\right]^{T}$.

\section{PROBLEM STATEMENT}

Consider a linear system, the dynamics of which are described by the equation

$$
M \ddot{p}+K \dot{p}+G p=u_{s}
$$


where $p(t) \in \mathbb{R}^{n}, \dot{p}(t) \in \mathbb{R}^{n}$ are the states, $u_{s}\left(u_{h}, u_{f}\right) \in \mathbb{R}^{n}$, denoting the external input, is a function of $u_{h}$ (the input applied by the operator) and $u_{f}$ (the input applied by the feedback controller), $M=M^{T}>0, K=K^{T} \geq 0$ and $G=G^{T}$. Note that the class of linear systems (1) models linear mechanical system in which $M, K$ and $G$ are the inertia matrix, the Coulomb friction coefficient and the potential energy matrix, respectively. Together with equation (1) we consider an output signal

$$
y=C\left[\begin{array}{c}
p \\
\dot{p}
\end{array}\right]+D u_{s}
$$

where $C$ is such that the system (1)-(2) is observable. In particular we consider two special cases: $y(t) \in \mathbb{R}^{2 n}, C=$ $I, D=0$ and $y(t) \in \mathbb{R}^{2 n}, C=\left[\begin{array}{ll}I & 0 \\ 0 & 0\end{array}\right], D=\left[\begin{array}{l}0 \\ I\end{array}\right]$.

Suppose $\mathcal{P}_{a}$ is a given and compact admissible configuration set for the state of the system (1) and $u_{h}$ is a given human input. Then the design of the shared-control is to find (if possible) a feedback controller, a safe subset and a sharing function such that the following properties hold: 1) the configuration of the system stays in $\mathcal{P}_{a}$ at all times; 2) $u_{s}$ does not change the aim of the human operator; 3) $u_{s}=u_{h}$ if the state of the system stays in the safe subset.

Assumption 1: We assume the non-empty admissible set $\mathcal{P}_{a}$ is defined by a group of linear inequalities, namely

$$
\mathcal{P}_{a}=\left\{p \in \mathbb{R}^{n} \mid S p+T \leq 0\right\},
$$

where $S=\left[s_{1}^{T}, s_{2}^{T}, \ldots, s_{m}^{T}\right]^{T} \in \mathbb{R}^{m \times n}, T=$ $\left[t_{1}, t_{2}, \ldots, t_{m}\right]^{T} \in \mathbb{R}^{m}$, with $s_{i} \in \mathbb{R}^{n}$ and $t_{i} \in \mathbb{R}$ for all $i \in\{1,2, \ldots, m\}$. In addition, If $m>n$ then for all $l \in[n+1, m]$ and $r_{1}, r_{2}, \ldots, r_{l} \in\{1,2, \ldots, m\}$ the matrices $S$ and $T$ satisfy the condition $\operatorname{rank}\left(\left[s_{r_{1}}^{T}, \ldots, s_{r_{l}}^{T}\right]^{T}\right)<$ $\operatorname{rank}\left(\left[h_{r_{1}}^{T}, \ldots, h_{r_{l}}^{T}\right]^{T}\right)$ where $h_{r_{i}}=\left[s_{r_{i}}, t_{r_{i}}\right]$ for all $i \in$ $\left\{r_{1}, \ldots, r_{l}\right\}$.

Definition 1: The $j^{\text {th }}$ constraint is said to be active for some velocity $\dot{p} \in \mathbb{R}^{n}$ if there exists $k>0$ such that $s_{j}(p+k \dot{p})+$ $t_{j}=0$.

Lemma 1: Consider the set $\mathcal{P}_{a}$ in (3) and assume Assumption 1 holds. Then for any fixed $\dot{p} \in \mathbb{R}^{n}$ no more than $n$ constraints are active.

Proof: If $m \leq n$ then the claim trivially holds. Consider now the case $m>n$. We prove the claim by contradiction. Suppose that, for a fixed $\dot{p},(n+1)$ constraints are active. Without loss of generality assume that these are the first $n+1$ constraints. Then $\left[s_{1}^{T}, \ldots, s_{n+1}^{T}\right]^{T}(p+k \dot{p})+\left[t_{1}, \ldots, t_{n+1}\right]^{T}=$ 0 . By Assumption 1 the above equation does not have any solutions, hence the claim.

By Lemma 1 the $m$ linear inequality constraints can be arranged into $N_{c}$ groups, where in general $N_{c} \leq\left(\begin{array}{c}m \\ n\end{array}\right)$ if $m \geq n$ and $N_{c}=1$ if $m<n$. In each group there are $n$ constraints. This is obvious if $m \geq n$ due to Lemma 1 . The statement that 'each group contains $n$ constraints' is without loss of generality if $m<n$ as discussed in [13].
Definition 2: The overall state space can be divided into three subspaces, the safe set $\mathcal{R}_{s}$, the hysteresis set $\mathcal{R}_{h}$ and the dangerous set $\mathcal{R}_{d}$, based on the distance and velocity towards the boundary. Relative to the $i^{\text {th }}$ group of active constraints,

$$
x^{i}=S^{i} p+T^{i} \leq 0,
$$

with $S^{i}$ invertible and $T^{i} \in \mathbb{R}^{n}$, the definitions of the safe, hysteresis and dangerous sets are given by equations (5) at the top of the next page, where $\mathcal{X}_{a}^{i}=S^{i} \mathcal{P}_{a}+T^{i}$, and $b_{2}>b_{1}>0$.

The following example is used to give an intuitive description of the definitions of the three subsets. Consider system (1) with $n=2$. Assume the admissible set is a square described by the constraints $\left[a_{1}^{T}, a_{2}^{T}, a_{3}^{T}, a_{4}^{T}\right]^{T} p+[-3-3-3-3]^{T} \leq 0$ where $a_{1}=[-1,0], a_{2}=[1,0], a_{3}=[0,-1]$ and $a_{4}=[0,1]$. Figure 1 provides illustrations of the sets $\mathcal{R}_{s}$ for different values of the velocity. In particular, the regions colored by green, yellow and red represent the safe, the hysteresis and the dangerous regions, respectively, for the values of the velocities indicated above each single subfigure.

Definition 3: The s-closed-loop and h-closed-loop systems are the system described by (1) and $M \ddot{p}(t)+K \dot{p}(t)+G p(t)=u_{h}$, respectively. In addition, $\Omega_{h}$ and $\Omega_{s}$ are used to denote the $\Omega$ limit set of the h-closed-loop and s-closed-loop, respectively.

\section{DESIGN OF THE FEEDBACK CONTROLLER}

In this section we provide a feedback control design for the two cases discussed in Section II with $\mathcal{P}_{a}$ defined in (3).

\section{A. Design of the Full State Feedback Controller}

Consider the $i^{\text {th }}$ group of constraints described by (4). The reference signal $x_{r}^{i}$, relative to $x^{i}$, is given by $x_{r}^{i}=$ $\left[x_{r_{1}}^{i}, x_{r_{2}}^{i}, \ldots, x_{r_{n}}^{i}\right]^{T}$, with $x_{r_{j}}^{i}$ defined by

$$
x_{r_{j}}^{i}= \begin{cases}-\epsilon, & \text { if } m \geq-\epsilon, \\ m\left[1-e^{\gamma(m+\epsilon)],}\right. & \text { if } m<-\epsilon,\end{cases}
$$

where $m=s_{j}^{i} p_{d}+t_{j}^{i}, \gamma>0, \epsilon$ is a sufficiently small positive constant and $p_{d}$ denotes the reference configuration for the variable $p$. In the trajectory tracking case, the feedback controller shares the same information of the reference signal as the human, hence $p_{d}$ is known to the feedback controller. Otherwise, in the free-driving case, $p_{d}(t)$ is calculated from $u_{h}(t)$. Note that $x_{r_{j}}^{i}$ is a smooth function with all negative values. As a result, $\dot{x}_{r}^{i}$ exists and

$$
p_{r}^{i}(t)=S^{i^{-1}}\left(x_{r}^{i}(t)-T^{i}\right), \quad v_{r}^{i}(t)=S^{i^{-1}} \dot{x}_{r}^{i}(t) .
$$

Note that $\left(p_{r}^{i}, v_{r}^{i}\right) \in \mathcal{P}_{a} \times \mathbb{R}^{n}$. Using equation (1), it is possible to calculate the reference input $u_{r}^{i}$ related to the $i^{\text {th }}$ groups of constraints, namely

$$
u_{r}^{i}=M \dot{v}_{r}^{i}+K v_{r}^{i}+G p_{r}^{i}
$$

Finally, the state feedback controller $u_{s f}^{i}$ is designed by standard back-stepping method [18] to make the derivative 


$$
\begin{gathered}
\tilde{\mathcal{R}}_{s}^{i}=\left\{\left(x^{i}, \dot{x}^{i}\right) \in \mathcal{X}_{a}^{i} \times \mathbb{R}^{n}: \dot{x}_{j}^{i} \leq \frac{1}{x_{j}^{i}+b_{2}}-\frac{1}{b_{2}} \text { if } x_{j}^{i} \geq-b_{2} \text { for all } j \in\{1,2, \ldots, n\}\right\} \\
\tilde{\mathcal{R}}_{h}^{i}=\left\{\begin{array}{l}
\left.\left(x^{i}, \dot{x}^{i}\right) \in \mathcal{X}_{a}^{i} \times \mathbb{R}^{n}: \exists j \in\{1,2, \ldots, n\} \text { such that } \dot{x}_{j}^{i}>\frac{1}{x_{j}^{i}+b_{2}}-\frac{1}{b_{2}} \text { and } x_{j}^{i} \geq-b_{2}\right\} \\
\text { and } \dot{x}_{k}^{i}<\frac{1}{x_{k}^{i}+b_{1}}-\frac{1}{b_{1}} \text { if } x_{k}^{i} \geq-b_{1} \text { for all } k \in\{1,2, \ldots, n\}
\end{array}\right. \\
\tilde{\mathcal{R}}_{d}^{i}=\left\{\left(x^{i}, \dot{x}^{i}\right) \in \mathcal{X}_{a}^{i} \times \mathbb{R}^{n}: \exists j \in\{1,2, \ldots, n\} \text { such that } \dot{x}_{j}^{i} \geq \frac{1}{x_{j}^{i}+b_{1}}-\frac{1}{b_{1}} \text { and }-b_{1} \leq x_{j}^{i}<0\right\}
\end{gathered}
$$
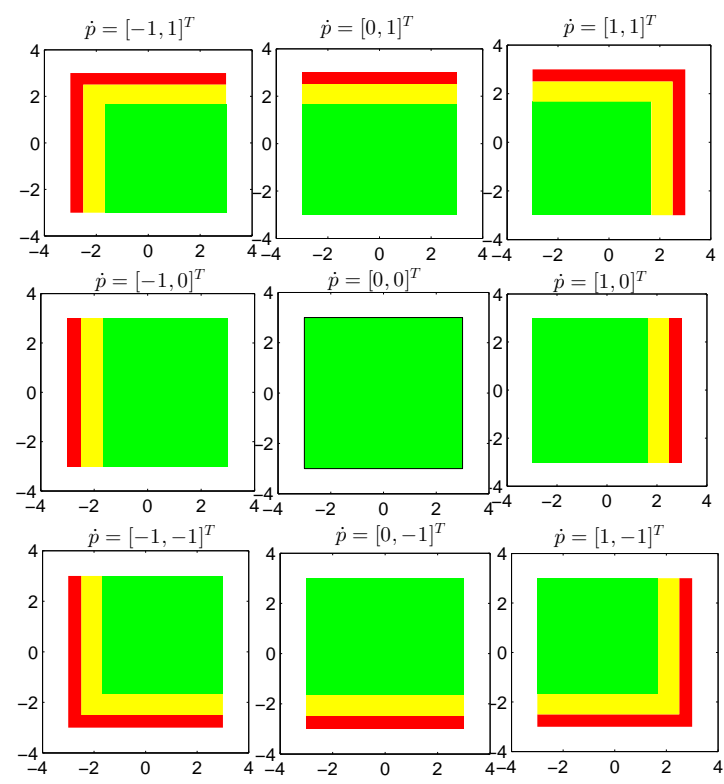

Fig. 1. Illustration of the safe, the hysteresis and the dangerous sets for the case $n=2\left(b_{1}=1, b_{2}=2\right)$.

of the Lyapunov function (13) negative definite and it is given by

$$
\begin{aligned}
u_{s f}^{i}=M\left(\dot{v}_{r}^{i}+\dot{v}_{e}^{i *}-S^{i}\left[\begin{array}{c}
\log \frac{s_{1}^{i} p+t_{1}^{i}}{s_{1}^{i} p_{r}^{i}+t_{1}^{i}} \\
s_{1}^{i} p+t_{1}^{i} \\
\vdots \\
\log \frac{s_{n}^{i} p+t_{n}^{i}}{s_{n}^{i} p_{r}^{i}+t_{n}^{i}} \\
\frac{s_{n}^{i} p+t_{n}^{i}}{n}
\end{array}\right]\right) \\
+K\left(v_{e}^{i}+v_{r}^{i}\right)+G\left(p_{e}^{i}+p_{r}^{i}\right)-\eta M\left(v_{e}^{i}-v_{e}^{i *}\right)
\end{aligned}
$$

where $\eta>0, v_{e}^{i}=\dot{p}-v_{r}^{i}, p_{e}^{i}=p-p_{r}^{i}$ and

$$
v_{e}^{i *}=S^{i-1}\left[\begin{array}{c}
\log \frac{s_{1}^{i} p+t_{1}^{i}}{s_{1}^{i} p_{r}^{i}+t_{1}^{i}} \\
\vdots \\
\log \frac{s_{n}^{i} p+t_{n}^{i}}{s_{n}^{i} p_{r}^{i}+t_{n}^{i}}
\end{array}\right]+\left[\begin{array}{c}
\frac{s_{1}^{i} p+t_{1}^{i}}{s_{1}^{i} p_{r}^{i}+t_{1}^{i}} v_{r_{1}}^{i}-v_{r_{1}}^{i} \\
\vdots \\
\frac{s_{n}^{i} p+t_{n}^{i}}{s_{n}^{i} p_{r}^{i}+t_{n}^{i}} v_{r_{n}}^{i}-v_{r_{n}}^{i}
\end{array}\right]
$$

\section{B. Design of the Partial State Feedback Controller}

This section discusses the design of the feedback controller in the case not all states are measurable. The first step is to design an observer for the system which is obtained from the measurements of $u_{s}$ and $y$, via a dynamical system of the form

$$
\left[\begin{array}{c}
\dot{\hat{p}} \\
\dot{\hat{v}}
\end{array}\right]=A\left[\begin{array}{c}
\hat{p} \\
\hat{v}
\end{array}\right]+B u_{s}+H y
$$

Let $A=\left[\begin{array}{cc}0 & I \\ -M^{-1} G & -M^{-1} K\end{array}\right]-H C, B=\left[\begin{array}{c}0 \\ M^{-1}\end{array}\right]-$ $H D$, where $H$ is such that $\lambda(A) \subset \mathbb{C}^{-}$. Suppose $\hat{p}$ and $\hat{v}$ are estimates of $p$ and $\dot{p}$, respectively, and define the estimation error $e(t)$ as $e(t)=\left[e_{p}(t), e_{v}(t)\right]^{T}=[p(t)-\hat{p}(t), \dot{p}(t)-\hat{v}(t)]^{T}$. Then the system described by the equation $\dot{e}=A e$ has an exponentially stable equilibrium at $e=0$, i.e. there exist positive constants $c, \gamma$ and $\delta$ such that if $\|e(0)\|_{2}<\delta$, then $\|e(t)\|_{2}<c e^{-\gamma t}\|e(0)\|_{2}$ for all $t \geq 0$. Furthermore, there exists $\alpha>0$ and $\beta>0$ such that $\left|e_{i}(t)\right| \leq \mathscr{E}$ for all $i \in\{1,2, \ldots, 2 n\}$, where $\mathscr{E}=\alpha e^{-\beta t} \max _{1 \leq i \leq 2 n}\left|e_{i}(0)\right|$. Similarly to the design of the state feedback controller, we design the partial state feedback controller in the case $m=n$. Consider the $i^{\text {th }}$ group of constraints described by (4) and let

$$
p_{r}^{i}=\mathcal{S}^{i-1}\left(x_{r}^{i}-\mathcal{T}^{i}-\mathscr{E}\left|\mathcal{S}^{i} a\right|\right), v_{r}^{i}=\mathcal{S}^{i-1}\left(\dot{x}_{r}^{i}+\beta \mathscr{E}\left|\mathcal{S}^{i} a\right|\right),
$$

where $a=[1,1, \ldots, 1]^{T}$ and $x_{r}^{i}$ is given by (6). Then the position feedback controller relative to the $i^{\text {th }}$ group of active constraints is given by

$$
\begin{aligned}
u_{o f}^{i}= & -\eta M\left(\hat{v}-v_{r}^{i}-\hat{v}_{e}^{i *}\right)+K\left(\hat{v}-v_{r}^{i}\right)+G\left(\hat{p}-p_{r}^{i}\right) \\
+M\left(\dot{\hat{v}}_{e}^{i *}-S^{i^{T}}\right. & {\left.\left[\begin{array}{c}
\frac{\log \frac{s_{1}^{i} \hat{p}+t_{1}^{i}+\mathscr{E}\left|s_{1}^{i} a\right|}{s_{1}^{i} p_{r}^{i}+t_{1}^{i}}}{s_{1}^{i} \hat{p}+t_{1}^{i}+\mathscr{E}\left|s_{1}^{i} a\right|} \\
\ldots \\
\log \frac{s_{n}^{i} \hat{p}+t_{n}^{i}+\mathscr{E}\left|s_{n}^{i} a\right|}{s_{n}^{i} p_{r}^{i}+t_{n}^{i}} \\
\frac{s_{n}^{i} \hat{p}+t_{n}^{i}+\mathscr{E}\left|s_{n}^{i} a\right|}{}
\end{array}\right]\right), }
\end{aligned}
$$


where $\hat{v}_{e}^{i *}=\gamma \mathscr{E} a+\mathcal{S}^{i}-1\left[\begin{array}{c}\frac{\log \frac{s_{1}^{i} \hat{p}+t_{1}^{i}+\mathscr{E}\left|s_{1}^{i} a\right|}{s_{1}^{i} p_{r}^{i}+t_{1}^{i}}}{s_{1}^{i} \hat{p}+t_{1}^{i}+\mathscr{E}\left|s_{1}^{i} a\right|} \\ \ldots \\ \log \frac{s_{n}^{i} \hat{p}+t_{n}^{i}+\mathscr{E}\left|s_{n}^{i} a\right|}{s_{n}^{i} p_{r}^{i}+t_{n}^{i}} \\ \frac{s_{n}^{i} \hat{p}+t_{n}^{i}+\mathscr{E}\left|s_{n}^{i} a\right|}{\mathrm{s}_{\mathrm{n}} \hat{\mathrm{p}}+\mathrm{t}_{\mathrm{n}}^{\mathrm{i}}+\mathscr{E}\left|\mathrm{s}_{\mathrm{n}}^{1} \mathrm{a}\right|}\end{array}\right]+$
$\operatorname{diag}\left(\frac{\mathrm{s}_{\mathrm{n}}^{\mathrm{i}} \hat{\mathrm{p}}+\mathrm{t}_{1}^{\mathrm{i}}+\mathrm{t}_{\mathrm{n}}^{\mathrm{i}}+\mathscr{E}\left|\mathrm{s}_{1}^{\mathrm{i}} \mathrm{a}\right|}{\mathrm{s}_{1}^{\mathrm{i}} \mathrm{p}_{\mathrm{r}}^{\mathrm{i}}+\mathrm{t}_{1}^{\mathrm{i}}}-1, \ldots, \mathrm{v}_{\mathrm{r}}^{\mathrm{i}}\right.$.

We now present a preliminary result.

\section{Lemma 2: Consider}

- the system (1)-(2)-(10) with $u_{s}=u_{o f}^{i}$ given by (12), $p_{r}^{i}$ and $v_{r}^{i}$ given by (11), $u_{r}^{i}$ given by (8),

- the system (1) with $u_{s}=u_{s f}^{i}$ given by (9), $p_{r}^{i}$ and $v_{r}^{i}$ given by (7), $u_{r}^{i}$ given by (8).

Assume $p(0) \in \mathcal{P}_{a}$ and $S^{i} \hat{p}(0)+T^{i}+\mathscr{E}(0)\left|S^{i} a\right| \leq 0$ for all $i \in\left\{1,2, \ldots, N_{c}\right\}$. Then $\lim _{t \rightarrow \infty}\left(p(t)-p_{r}^{i}(t)\right)=0, \lim _{t \rightarrow \infty}(v(t)-$ $\left.v_{r}^{i}(t)\right)=0$ and $p(t) \in \mathcal{P}_{a}$ for all $t \geq 0$.

Proof: To begin with, we prove that the lemma holds for the system with state feedback. Consider the $i^{\text {th }}$ group of active constraints, let $z^{i}=\left(z_{1}^{i}, z_{2}^{i}, \ldots, z_{n}^{i}\right)^{T}$, with $z_{j}^{i}=$ $\log \frac{x_{j}^{i}}{x_{r_{j}}^{i}}$ for all $j \in\{1, \ldots, n\}$, where $x_{j}^{i}$ and $x_{r_{j}}^{i}$ are defined by (4) and (6), respectively. Consider the Lyapunov function candidate

$$
L^{i}\left(z^{i}, v_{e}^{i}\right)=\frac{1}{2}\left[z^{i^{T}} z^{i}+\left(v_{e}^{i}-v_{e}^{i *}\right)^{T}\left(v_{e}^{i}-v_{e}^{i *}\right)\right],
$$

and calculate its time derivative along the trajectories of the system. This yields $\dot{L}^{i}\left(z^{i}, v_{e}^{i}\right)=\left(\sum_{j=1}^{n} \frac{z_{j}^{i 2} \exp \left(-z_{j}^{i}\right)}{x_{r_{j}}^{i}}\right)-\eta\left(v_{e}^{i}-\right.$ $\left.v_{e}^{i *}\right)^{T}\left(v_{e}^{i}-v_{e}^{i *}\right)$. Therefore $L^{i}<0$ for all $z^{i} \neq 0$ and $v_{e}^{i} \neq 0$ since $x_{r_{j}}^{i}<0$, for all $j \in\{1,2, \ldots, n\}$. Consider now the switch from $u_{s f}^{i}$ to $u_{s f}^{j}$ with $i \neq j$. If the shared-control input $u_{s}$ switches directly from $u_{s f}^{i}$ to $u_{s f}^{j}$, then $L^{j}\left(t_{0}\right) \leq L^{i}\left(t_{0}\right)$ and $\dot{L}^{j}(t)<0$ for all $t \geq t_{0}$ and $(p, v) \neq\left(p_{r}, v_{r}\right)$. If $u_{s}$ switches not directly from $u_{s f}^{i}$ to $u_{s f}^{j}$, i.e. if $(p, v)$ leaves $\mathcal{R}_{d}^{i}$ at $t=t_{1}$ and enter $\mathcal{R}_{d}^{j}$ at $t=t_{2}$, then due to the existence of $\mathcal{R}_{h}^{i}, \exists \delta^{i} t>0$ such that $u_{s}(t)=u_{s f}^{i}(t)$ for all $t \in\left[t_{1}, t_{1}+\delta^{i} t\right]$. Let $\Delta t=\min _{i=1}^{N_{c}} \delta^{i} t$ and note that $\Delta>0$. Then there always exists $\eta>0$ such that $\int_{t_{0}}^{t_{0}+\Delta t} \dot{L}^{j} d t \leq L^{j}\left(t_{2}\right)-L^{i}\left(t_{1}\right)$, i.e. $L^{j}\left(t_{2}+\Delta t\right) \leq L^{i}\left(t_{1}\right)$. Let $\left\{i_{1}, i_{2}, \ldots, i_{I}\right\}$ be a sequence of active group of constraints, where $i_{j} \in\left\{1,2, \ldots, N_{c}\right\}$ for all $j \in\{1,2, \ldots, I\}$, and the $i_{j}^{\text {th }}$ group is active for the time period $\left(t_{i_{j}}, T_{i_{j}}\right]$ with $t_{i_{j+1}}=T_{i_{j}}$ for all $j \in\{1,2, \ldots, I-1\}$. Therefore $0 \leq L^{i_{I}}\left(T_{i_{I}}\right)<\cdots<L^{i_{2}}\left(T_{i_{2}}\right)<L^{i_{1}}\left(T_{i_{1}}\right)$. Define the overall Lyapunov function $L(t)$ as $L(t)=L^{i_{j}}(t)$, if $t \in$ $\left(t_{i_{j}}, T_{i_{j}}\right]$. From the above analysis $L(t)$ is a multiple Lyapunov function and this implies the first claim. Furthermore, $x^{i}(t) \leq$ $-\epsilon<0$ for all $t \geq 0$ by definition of $z^{i}$. Hence, $p(t) \in \mathcal{P}_{a}$ for all $t \geq 0$.

The proof of the lemma for the system with partial state feedback is similar to that given above. A slight modification is needed for the definitions of $x^{i}$ and $L^{i}$, i.e. $x^{i}=S^{i} \hat{p}+T^{i}+$
$\mathscr{E}\left|S^{i} a\right|$ and $L^{i}\left(z^{i}, \hat{v}_{e}^{i}\right)=\frac{1}{2}\left[z^{i T} z^{i}+\left(\hat{v}_{e}^{i}-\hat{v}_{e}^{i *}\right)^{T}\left(\hat{v}_{e}^{i}-\hat{v}_{e}^{i *}\right)\right]$, where $\hat{v}_{e}^{i}=\hat{v}-v_{r}^{i}$. The derivative of $L^{i}$ is calculated as $\dot{L}^{i}\left(z^{i}, \hat{v}_{e}^{i}\right)=\left(\sum_{j=1}^{n} \frac{z_{j}^{i 2} \exp \left(-z_{j}^{i}\right)}{x_{r_{j}}^{i}}\right)-\eta\left(\hat{v}_{e}^{i}-\hat{v}_{e}^{i *}\right)^{T}\left(\hat{v}_{e}^{i}-\hat{v}_{e}^{i *}\right)$, hence $\lim _{t \rightarrow \infty}\left(p(t)-p_{r}^{i}(t)\right)=0$ and $\lim _{t \rightarrow \infty}\left(v(t)-v_{r}^{i}(t)\right)=0$. $p(t) \in \stackrel{\mathcal{P}}{\text { Pa }}_{a}^{\infty}$ for all $t \geq 0$ is a consequence of the definition of $z^{i}$.

\section{DESIGN OF THE SHARED-CONTROL LAW}

This section gives shared-control laws for system (1) with full state feedback and partial state feedback, respectively.

\section{A. Shared-Control Law with Full State feedback}

With reference to the $i^{\text {th }}$ group of $n$ constraints, the state space can be partitioned into three subsets by the equations (5). To eliminate ambiguity for different groups of constraints, it is essential to "push" the subsets back into the $(p, \dot{p})$ coordinates using the relations $\mathcal{R}_{\alpha}^{i}=\operatorname{diag}\left(\mathrm{S}^{\mathrm{i}^{-1}}, \mathrm{~S}^{\mathrm{i}-1}\right)\left(\tilde{\mathcal{R}}_{\alpha}^{\mathrm{i}}-\operatorname{col}\left(\mathrm{T}^{\mathrm{i}}, 0\right)\right)$, where $\alpha=\{s, h, d\}$. Note that, by construction, $\mathcal{R}_{s}^{i} \cup \mathcal{R}_{h}^{i} \cup$ $\mathcal{R}_{d}^{i}=\mathcal{R}$ for all $i \in\left\{1,2, \ldots, N_{c}\right\}$, which indicates that for any fixed $\dot{p}$ the union of the safe, the hysteresis and the dangerous set relative to the $i^{\text {th }}$ group of active constraints coincides with the overall feasible state space. The overall safe, hysteresis and dangerous set for different groups of constraints are then defined as $\mathcal{R}_{d}=\mathcal{R}_{d}^{1} \cup \cdots \cup \mathcal{R}_{d}^{N_{c}}, \mathcal{R}_{h}=$ $\mathcal{R}_{h}^{1} \cup \cdots \cup \mathcal{R}_{h}^{N_{c}}, \mathcal{R}_{s}=\mathcal{R}_{s}^{1} \cap \cdots \cap \mathcal{R}_{s}^{N_{c}}$, and they have the following property by construction: $\mathcal{R}=\mathcal{R}_{d} \cup \mathcal{R}_{h} \cup \mathcal{R}_{s}$.

On the basis of these subsets, the state-feedback shared-control law is then defined, similarly to [15], as

$$
u_{s}=\left(\min _{1 \leq i \leq N_{c}} k_{s f}^{i}\right) u_{h}+\sum_{i=1}^{N_{c}}\left[\left(1-k_{s f}^{i}\right) u_{s f}^{i}\right],
$$

where $^{1}$

$k_{s f}^{i}(p, v, t)= \begin{cases}0, & (p, v) \in \mathcal{R}_{d}^{i} \text { and } L^{i}=\min _{j=I_{1}}^{I_{n_{c}}} L^{j}, \\ l_{s f}^{i}(p, v, t), & (p, v) \in \mathcal{R}_{h}^{i}, \\ 1, & \text { otherwise, }\end{cases}$ with $l_{s f}^{i}(p, v, t)= \begin{cases}0, & \text { if } k_{s f}^{i}\left(t^{-}\right)=0 \\ 1, & \text { if } k_{s f}^{i}\left(t^{-}\right)=1\end{cases}$

\section{B. Shared-Control Law with Partial State Feedback}

Consistently with Section IV-A, relative to the $i^{\text {th }}$ group of $n$ constraints, the feasible state space can be partitioned into three subsets as in (5), with $x^{i}=S^{i} \hat{p}+T^{i}+\mathscr{E}\left|S^{i} a\right|$ and $\mathcal{X}_{a}^{i}=S^{i} \mathcal{P}_{a}+T^{i}+\mathscr{E}\left|S^{i} a\right|$. To use a uniform set of coordinates for different groups of constraints, we pull the subsets back into the $(\hat{p}, \hat{v})$ coordinates. This can be done using the equation $\mathcal{R}_{\alpha}^{i}=\operatorname{diag}\left(\mathrm{S}^{\mathrm{i}-1}, \mathrm{~S}^{\mathrm{i}^{-1}}\right)\left(\tilde{\mathcal{R}}_{\alpha}^{\mathrm{i}}-\operatorname{col}\left(\mathrm{T}^{\mathrm{i}}, 0\right)+\right.$ $\left.\operatorname{diag}\left(\mathrm{S}^{\mathrm{i}^{-1}}, \mathrm{~S}^{\mathrm{i}^{-1}}\right) \operatorname{col}(-\mathscr{E} \mathrm{a}, \beta \mathscr{E} \mathrm{a})\right)$, where $\alpha=\{s, h, d\}$. Note

\footnotetext{
${ }^{1}$ Note that $L^{j}$ is defined in equation (13).
} 
that these sets have the same properties as those stated in Section IV-A. The partial state feedback shared-control law is then defined as

$$
u_{s}=\left(\min _{1 \leq i \leq N_{c}} k_{o f}^{i}\right) u_{h}+\sum_{i=1}^{N_{c}}\left[\left(1-k_{o f}^{i}\right) u_{o f}^{i}\right],
$$

where

$k_{o f}^{i}(\hat{p}, \hat{v}, t)= \begin{cases}0, & (\hat{p}, \hat{v}) \in \mathcal{R}_{d}^{i} \text { and } L^{i}=\min _{j=I_{1}}^{I_{n_{c}}} L^{j}, \\ l_{o f}^{i}(\hat{p}, \hat{v}, t), & (\hat{p}, \hat{v}) \in \mathcal{R}_{h}^{i}, \\ 1, & \text { otherwise, }\end{cases}$ with $l_{o f}^{i}(\hat{p}, \hat{v}, t)=\left\{\begin{array}{ll}0, & \text { if } k_{o f}^{i}\left(t^{-}\right)=0, \\ 1, & \text { if } k_{o f}^{i}\left(t^{-}\right)=1,\end{array}\right.$ and $I_{1}, I_{2}, \ldots, I_{n_{c}}$ is defined as: $(\hat{p}, \hat{v}) \in \mathcal{R}_{d}^{I_{1}} \cap \mathcal{R}_{d}^{I_{2}} \cap \cdots \cap \mathcal{R}_{d}^{I_{n_{c}}}$.

Lemma 3: Consider the system (1) with the shared-control input $u_{s}$ given by (9)-(14) and $(p(0), \dot{p}(0)) \in \mathcal{R}_{s}$. Suppose there exists $\tilde{t}>0$ such that $p(\tilde{t}) \notin \mathcal{P}_{a}$. Then there exists $0<t_{d}<\tilde{t}$ such that $\left(p\left(t_{d}\right), v\left(t_{d}\right)\right) \in \mathcal{R}_{d}$.

Theorem 1: Consider

- the system (1)-(2)-(10) with the shared-control input $u_{s}$ given by (12)-(15),

- the system (1) with the shared-control input $u_{s}$ given by (9)-(14).

Assume $p(0) \in \mathcal{P}_{a}$ and $S^{i} \hat{p}(0)+T^{i}+\mathscr{E}(0)\left|S^{i} a\right| \leq 0$ for all $i \in$ $\left\{1,2, \ldots, N_{c}\right\}$. Then there exists $\eta>0$ and $b_{2}>b_{1}>0$ such that the s-closed-loop system has the following properties.

1) $p(t) \in \mathcal{P}_{a}$ for all $t \geq 0$.

2) $\Omega_{s}=\Pi_{\mathcal{R}_{s}}\left(\Omega_{h}\right)$.

3) $u_{s}(t)=u_{h}(t)$ for all $t \geq 0$ and $(\hat{p}(t), \hat{v}(t)) \in \mathcal{R}_{s} \backslash \mathcal{R}_{d}$ (in the case of partial state feedback) or $(p(t), \dot{p}(t)) \in$ $\mathcal{R}_{s} \backslash \mathcal{R}_{d}$ (in the case of full state feedback).

Proof: We prove the properties hold for the system with state feedback since the proof is similar for the system with partial state feedback. As Lemma 2 states, the feedback controller (either $u_{s f}^{i}$ or $u_{o f}^{i}$ ) is such that the state $p$ of the system for all trajectories remain in $\mathcal{P}_{a}$. In addition, Lemma 3 states that any trajectory exiting $\mathcal{R}$ should first enter $\mathcal{R}_{d}$, where the feedback controller is active. As a result, the set $\mathcal{R}$ is forward invariant, hence claim 1) holds. If $\Omega_{h} \subset \mathcal{R}_{s}$, then claim 2) is a consequence of the general results in [15], and of the fact that $\Omega_{h}$ is the $\Omega$-limit set of both the hclosed-loop and the s-closed-loop systems (by assumption, the former, and by the design of the shared-control law together with the feedback controller, the latter). Otherwise, as detailed in the Proof of Lemma 2, the $\Omega$-limit set of the closed-loop system with the feedback-controller is $\Pi_{\mathcal{R}_{s}}\left(\Omega_{h}\right)$. In addition, Lemma 3 indicates that the trajectory of the system enters $\mathcal{R}_{d}$ where the feedback controller is active, hence driving the state of the system back to $\mathcal{R}_{s}$ before leaving the admissible set $\mathcal{R}$. Therefore, claim 2) holds. Finally, claim 3) is a direct consequence of the definition of the shared-control law.

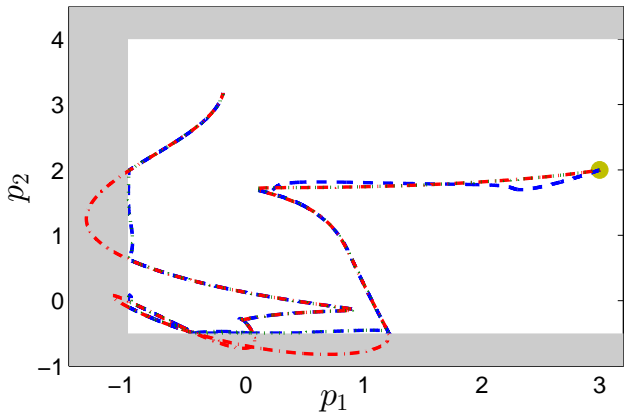

Fig. 2. Paths in the $\left(p_{1}, p_{2}\right)$-plane of the system (16) for the set $\mathcal{P}_{a}$ given by (17): h-closed-loop with state-feedback (red, dash-dotted), s-closed-loop with state-feedback (green, dotted) and s-closed-loop with output-feedback (blue, dashed). The green, large, dot denotes the initial position.

Remark 1: As detailed in [14], the shared-control algorithms presented above can also be used for noncovex feasible regions defined by linear inequalities complemented with logic "statements".

\section{NUMERICAL EXAMPLES}

This section provides two numerical examples. Note that the admissible set for the second case is non-convex: this is used to illustrate the discussion in Remark 1. Further case studies can be found in [13] and [14].

\section{A. Convex $\mathcal{P}_{a}$}

Consider a two degrees-of-freedom, linear system described by the equations

$$
\begin{aligned}
& \dot{p}_{1}=v_{1}, \\
& \dot{p}_{2}=v_{2}, \\
& \dot{v}_{1}=-p_{1}-0.5 p_{2}-v_{1}-0.3 v_{2}+u_{1}, \\
& \dot{v}_{2}=-0.4 p_{1}-2 p_{2}-0.3 v_{1}+0.5 v_{2}+u_{2} .
\end{aligned}
$$

Assume the admissible set $\mathcal{P}_{a}$ is defined by

$$
\mathcal{P}_{a}=\left\{p=\left[p_{1}, p_{2}\right]^{T} \mid p_{1} \geq-1,-0.5 \leq p_{2} \leq 4\right\}
$$

Let $u_{h}=\left[u_{h_{1}}, u_{h_{2}}\right]^{T}$, where $u_{h_{i}} \in[-1,1]$ for $i \in\{1,2\}$, models the action of the human operator who "drives" the system in the set $\mathcal{P}_{a}$. This models for example the system driven by a joystick: $u_{h_{1}}=1$ and $u_{h_{1}}=-1$ refer to the joystick in the rightmost and leftmost position, respectively; $u_{h_{2}}=1$ and $u_{h_{2}}=-1$ refer to the joystick in the uppermost and lowermost position, respectively. We also assume that the position of the joystick relates to the human-exerted action on the system. Simulation results are given in Figure 2. It shows that the path resulting from the h-closed-loop enters the non-admissible region (gray, shaded), while the paths of the s-closed-loop remain in $\mathcal{P}_{a}$, thus indicating the effectiveness of the shared-control law. If the trajectory of the h-closed-loop system remains inside $\mathcal{P}_{a}$, then the trajectories of the s-closedloop system coincide with that of the h-closed-loop system. Otherwise, the $\left(p_{1}, p_{2}\right)$-path of the s-closed-loop system moves 


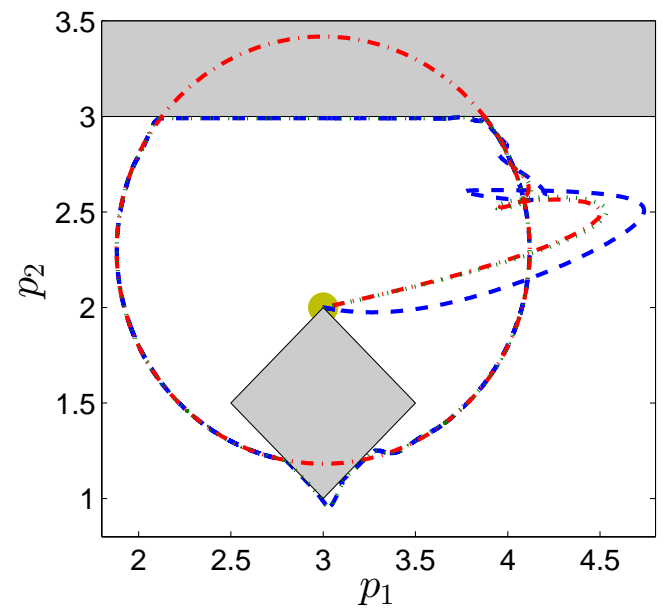

Fig. 3. Paths in the $\left(p_{1}, p_{2}\right)$-plane of the system (16) for the set $\mathcal{P}_{a}$ given by (18): h-control with state-feedback (red, dash-dotted), s-control with statefeedback (green, dotted) and s-control with output-feedback (blue, dashed). The green, large, dot denotes the initial position.

along the boundary of $\mathcal{P}_{a}$. This is consistent with claims (2) in Theorem 1. Note that in Figure 2 the blue, dashed, curve does not coincide with the green, dotted, curve because of the estimation error caused by the observer. However, the two curves asymptotically overlap since the estimation error converges to zero as Section III-B indicates.

\section{B. Non-Convex $\mathcal{P}_{a}$}

Consider again the system (16) and the concave admissible set $\mathcal{P}_{a}$ defined by

$$
\mathcal{P}_{a}=\left\{\begin{array}{l|r}
p_{2} \leq-p_{1}+4 \text { or } p_{2} \geq p_{1}-1 \\
\text { if } p_{1} \leq 3 \\
p=\left[p_{1}, p_{2}\right]^{T} & \begin{array}{r}
\text { or } p_{2} \geq-p_{1}+5 \\
\text { if } p_{1} \geq 3 \\
\text { and } p_{2} \leq 3
\end{array}
\end{array}\right\} .
$$

Suppose that the desired trajectory of the system is a circle centered at $(3,2.3)$ with radius $\sqrt{1.25}$. Simulation results are displayed in Figure 3. Note that even though the state $p$ is very close to the boundary of $\mathcal{P}_{a}$ at the beginning of the simulation, the $\left(p_{1}, p_{2}\right)$ path of the s-closed-loop system with full state feedback coincides with that of the h-closed-loop system because the $\mathrm{h}$-control drives the system states away from the boundary of $\mathcal{P}_{a}$.

\section{CONCLUSIONS}

We have presented a solution to the shared-control problem for a class of linear systems via full state feedback and partial state feedback. A hysteresis element is built to combine the human input and the feedback control input. Even though the sharedcontroller is designed for convex admissible configuration sets, it can also be used for non-convex admissible sets. Simulation results given in Section $\mathrm{V}$ show the effectiveness of the sharedcontrol algorithm. Note that both the theoretical analysis and the simulations are based on the exact knowledge of the underlying model. Further work to deal with constraints on the input signal and model uncertainties are in progress.

\section{REFERENCES}

[1] T. Rofer and A. Lankenau, "Ensuring safe obstacle avoidance in a shared-control system," in Proc. of IEEE International Conference on Emerging Technologies and Factory Automation, vol. 1, Barcelona, 1999 , pp. $1405-1414$.

[2] L. Saleh, P. Chevrel, F. Claveau, J. Lafay, and F. Mars, "Shared steering control between a driver and an automation: Stability in the presence of driver behavior uncertainty," IEEE Trans. on Intelligent Transportation Systems, vol. 14, no. 2, pp. 974-983, 2013.

[3] G. Hwang and H. Hashimoto, "Development of a human-robot-shared controlled teletweezing system," IEEE Trans. on Control Systems Technology, vol. 15, no. 5, pp. 960-966, 2007.

[4] D. Powell and M. O'Malley, "Efficacy of shared-control guidance paradigms for robot-mediated training," in Proc. of IEEE Conference on World Haptics Conference, 2011, pp. 427-432.

[5] J. Borenstein and Y. Koren, "Real-time obstacle avoidance for fast mobile robots," IEEE Trans. on Systems, Man and Cybernetics, vol. 19, no. 5, pp. 1179-1187, 1989.

[6] _ " "The vector field histogram-fast obstacle-avoidance for mobile robots," IEEE Journal of Robotics and Automation, vol. 7, no. 3, pp. 278-288, 1991.

[7] Y. Koren and J. Borenstein, "Potential field methods and their inherent limitations for mobile robot navigation," in Proc. of IEEE Conference on Robotics and Automation, 1991, pp. 1398-1404.

[8] J. Kim, Y. Kim, Y. Kim, W. Kang, and J. An, "Design based on a shared look-up table for an obstacle avoidance fuzzy controller for mobile robots," in Proc. of Intelligent Systems Design and Applications, 2009, pp. $731-736$.

[9] E. Gilbert and I. Kolmanovsky, "Nonlinear tracking control in the presence of state and control constraints: a generalized reference governor," Automatica, vol. 38, pp. 2063-2073, 2002.

[10] K. Tee, S. Ge, and E. Tay, "Barrier Lyapunov functions for the control of output-constrained nonlinear systems," Automatica, vol. 45, pp. 918 927, 2009.

[11] M. Kimmel, Y. Zhang, and S. Hirche, "Invariance control with chattering reduction," in Proc. of IEEE Conference on Decision and Control, 2014, pp. 68-74.

[12] W. Ren and R. Beard, "Satisficing approach to human-in-the-loop safeguarded control," in Proc. of American Control Conference, 2005, pp. 4985-4990.

[13] J. Jiang and A. Astolfi, "Shared-control for fully actuated linear mechanical systems," in Proc. of IEEE Conference on Decision and Control, Florence, Italy, 2013, pp. 4699-4704.

[14] _ , "Output-feedback shared-control for fully actuated linear mechanical systems," in Proc. of American Control Conference, Portland, USA, 2014, pp. 335-340.

[15] C. Prieur, "Uniting local and global controllers with robustness to vanishing noise," Math. Control Signals Systems, vol. 14, pp. 143-172, 2001.

[16] L. Zaccarian, "Dynamic allocation for input redundant control systems," Automatica, vol. 45, no. 6, pp. 1431-1438, 2009.

[17] C. Prieur and A. Teel, "Uniting local and global output feedback controller," IEEE Trans. Automatic Control, vol. 56, no. 7, pp. 16361679, 2011.

[18] M. Krstic, P. Kokotovic, and I. Kanellakopoulos, Nonlinear and Adaptive Control Design. New York, NY, USA: John Wiley \& Sons, Inc., 1995. 